\title{
A Structured Generalization of Decode-and-Forward Strategies for Multiple-Relay Networks
}

\author{
Peyman Razaghi and Wei Yu \\ Department of Electrical and Computer Engineering, \\ University of Toronto, Canada \\ e-mails: \{peyman,weiyu\}@ comm.utoronto.ca
}

\begin{abstract}
This paper presents a structured characterization of a class of decode-and-forward (DF) strategies for arbitrary multirelay networks. In contrast to conventional DF strategies in which each relay transmits the bin index of the source message only, the proposed generalized DF strategies allow each relay to decode a selection of messages from other nodes and forward bin indices for them. A tree structure is utilized to characterize the dependencies of messages on each other. Based on this tree structure, closed-form expressions for the achievable rates of these DF schemes are derived. The proposed DF strategy improves the previous multirelay DF rates and gives the capacity of new forms of degraded multirelay networks.
\end{abstract}

\section{INTRODUCTION}

Relay networks have attracted extensive attention recently [1], [2], [3], [4], [5]. In a relay network, a source communicates with a destination with the help of one or more relays. One of the main strategies for the relay network is the decode-and-forward (DF) strategy, originally introduced in $[6$, Theorem 1]. In the DF strategy for the single-relay channel, the relay decodes the source message and forwards a bin index for it to the destination. However, in a multirelay network, many different DF schemes are possible, since each terminal may decode multiple messages from multiple upstream nodes and transmit multiple bin indices to multiple downstream nodes in a network.

This paper presents a structured formulation of a class of DF strategies for a given arbitrary multirelay network. This characterization is made possible via the use of a message tree which captures the dependencies between the messages of different terminals. Based on this tree structure, closedform expressions for the achievable rates of these DF schemes are formulated. This work is a generalization of [7] where a parity-forwarding protocol is proposed for two- and threerelay networks. The main contribution of this paper is a structured generalization of the parity forwarding protocol for an arbitrary network.

This work is one step toward a yet elusive theory of network information flow. Following an information theoretical approach, we formulate an improved set of achievable rates corresponding to various possible DF strategies for a given multirelay network. As a byproduct, the capacities of new forms of degraded multirelay networks are found.

\section{From Single-Relay DF to Multirelay DF}

The multirelay DF strategy devised in this paper is a generalization of the DF scheme of [6]. In the following, we outline how the single-relay DF scheme can be generalized to multirelays.

In the single-relay DF protocol, in each block, the source selects a new message $m_{0} \in\left\{1,2, \cdots, 2^{n R_{0}}\right\}$ of rate $R_{0}$. The set $\left\{1,2, \cdots, 2^{n R_{0}}\right\}$ is randomly binned into $2^{n R_{1}}$ bins of equal size. These bins are indexed by $m_{1} \in\left\{1,2, \cdots, 2^{n R_{1}}\right\}$. The relay decodes the source message in each block, and forwards its bin index $m_{1}$ in the next block. Since $m_{1}$ is computable from $m_{0}$, the source can broadcast the two messages $m_{0}$ and $m_{1}$ in each block, and cooperate with the relay in transmitting $m_{1}$. To encode the two messages $m_{0}$ and $m_{1}$, the source utilizes the superposition broadcast encoding scheme of the degraded broadcast channel [6, Chapter 14.6.2]. The source encodes $m_{0}$ on top of $m_{1}$ by constructing a random codebook for every codeword encoding $m_{1}$. Let $\mathbf{u}_{1}\left(m_{1}\right)$ denote the random codeword encoding $m_{1}$ generated according to the probability distribution (PDF) $p\left(u_{1}\right)$. The source generates a random codebook of size $2^{n R_{0}}$ indexed by $m_{0}$ for each sequence $\mathbf{u}_{1}\left(m_{1}\right)$ according to $p\left(u_{0} \mid u_{1}\right)$, i.e., for each $\mathbf{u}_{1}\left(m_{1}\right), 2^{n R_{0}}$ codewords $\mathbf{u}_{0}\left(m_{0} \mid m_{1}\right)$ are randomly generated according to $\Pi_{i} p\left(u_{0 i} \mid u_{1 i}\right)$ where $u_{0 i}$ and $u_{1 i}$ denote the $i$ th elements of $\mathbf{u}_{0}$ and $\mathbf{u}_{1}$, respectively.

A generalization of this single-relay scheme to a multirelay network can be obtained by allowing each relay to decode a selection of messages of other transmitters in each block, and to forward their corresponding bin indices in the next block. In such a scheme, a relay could transmit a bin index for a relay message, which itself is the bin index of yet another message. To characterize these message dependencies, a tree structure can be utilized in which messages are represented by the nodes and the child of a message represents its bin index.

As in the single-relay case, a broadcast strategy must be utilized in the multirelay network to encode multiple messages at the source or at the relays. Since each transmitter may be capable of determining the messages of subsequent relays, it can cooperate in transmitting this set of known messages. This paper considers multirelay DF protocols that utilize superposition broadcast encoding. Although the superposition broadcast strategy is restrictive in that the messages have to be ordered according to the nature of degradedness and parallel relaying is not considered, it allows for an analytical formulation of a 

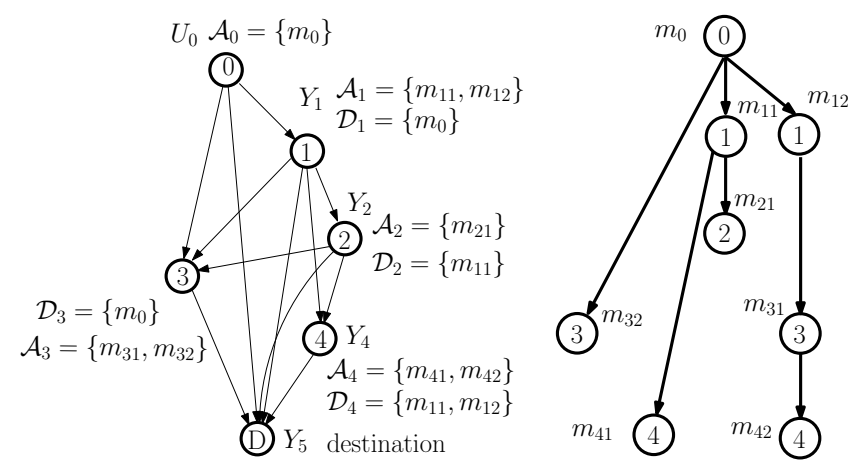

Fig. 1. Parity forwarding protocol for a four-relay network. The sets $\mathcal{A}_{k}$, $1 \leq k \leq 4$, indicate the messages of the $k$ th relay. The set $\mathcal{D}_{k}, 1 \leq k \leq 4$, indicates messages that the $k$ th relay decodes. The message tree describes the dependencies of messages on each other.

set of achievable rates for an arbitrary network, extending the previous results on multirelay decode-and-forward.

\section{MultiRelay Parity ForWARDing}

Consider a multirelay network consisting of a pair of source and destination and $K$ relay terminals numbered from 1 to $K$. Our strategy is to assign one or more messages to each relay terminal. Each relay message is a bin index (parity) for a message of another transmitter.

The bin index (parity) of a message is determined via parity generation function. Let $\mathcal{B}_{y}=\left\{\mathcal{S}_{1}, \mathcal{S}_{2}, \cdots, \mathcal{S}_{2^{n R_{y}}}\right\}$ be a uniform random partitioning of $\left\{1,2, \cdots, 2^{n R_{x}}\right\}$ into $2^{n R_{y}}$ bins $\mathcal{S}_{k}$ of size $2^{n\left(R_{x}-R_{y}\right)}$ indexed by $\mathcal{Y}=\left\{1,2, \cdots, 2^{n R_{y}}\right\}$. The parity generation function $P_{\mathcal{B}_{y}}(\cdot)$ returns the bin index of its argument with respect to $\mathcal{B}_{y}$, i.e., $v=P_{\mathcal{B}_{y}}(u)$ if and only if $u \in \mathcal{S}_{v}$.

Let $m_{0} \in\left\{1,2, \ldots, 2^{n R_{0}}\right\}$ denote the source message of rate $R_{0}$. In each block, the first relay decodes the source message and forwards a set of parity messages $\mathcal{A}_{1}=$ $\left\{m_{11}, m_{12}, \ldots, m_{1 n_{1}}\right\}$ for $m_{0}$ (in the next block). The second relay decodes a selection of the source and the first relay messages, and forwards a set of parity messages $\mathcal{A}_{2}=$ $\left\{m_{21}, m_{22}, \ldots, m_{2 n_{2}}\right\}$ for its decoded set of messages, and so on for other relay terminals. (See Fig. 1 for an example.) The tree structure defined in the following characterizes this procedure.

Definition 1 (Message Tree): For a relay network, a message tree is defined by a directed tree $\tau=(\mathcal{M}, \mathcal{E})$ where $\mathcal{M}$ denotes the set of nodes corresponding to all messages passed in the network, and $\mathcal{E}$ denotes the set of directed edges. The source message $m_{0}$ denotes the root node. All other nodes correspond to parity messages sent by the relay terminals. The $k$ th relay, $1 \leq k \leq K$, transmits $n_{k}>0$ messages $m_{k 1}, m_{k 2}, \ldots, m_{k n_{k}}$. The edge connecting $m_{k^{\prime} l^{\prime}}$ to $m_{k l}$ corresponds to the parity generation function $P_{\mathcal{B}_{k l}}(\cdot)$, which maps the message $m_{k^{\prime} l^{\prime}} \in\left\{1,2, \cdots, 2^{n R_{k^{\prime} l^{\prime}}}\right\}$ to its bin index $m_{k l} \in\left\{1,2, \cdots, 2^{n R_{k l}}\right\}$ with respect to $\mathcal{B}_{k l}$, a random uniform partitioning of $\left\{1,2, \cdots, 2^{n R_{k^{\prime} l^{\prime}}}\right\}$ into $2^{n R_{k l}}$ bins. The random partitioning sets $\mathcal{B}_{k l}, 1 \leq k \leq K, 1 \leq l \leq n_{k}$ are generated independently.
Notation $1(\rightarrow, \nrightarrow)$ : We use $m \rightarrow m^{\prime}$ to denote that the message $m^{\prime}$ is a descendent of the message $m$. The message $m^{\prime}$ is a descendent of $m$ if there is a path from the message $m$ to the message $m^{\prime}$ on the message tree, i.e., $m^{\prime}$ is a parity message for $m$, either directly or via other intermediate parity messages. A message is considered to be a descendent of itself, i.e., $\forall m \in \mathcal{M}, m \rightarrow m$. We write $m \nrightarrow m^{\prime}$ if $m \rightarrow m^{\prime}$ is not true. We write $\mathcal{F} \rightarrow m$ to denote that the message $m$ is a descendent of the set $\mathcal{F} \subset \mathcal{M}$. The message $m$ is a descendent of $\mathcal{F}$ if $\exists f \in \mathcal{F}$ such that $f \rightarrow m$. A set of messages $\mathcal{G} \subset \mathcal{M}$ is a descendent of $\mathcal{F}$ if $\mathcal{F} \rightarrow g, \forall g \in \mathcal{G}$, which is denoted by $\mathcal{F} \rightarrow \mathcal{G}$. The " $\nrightarrow$ " relation for sets of messages denotes the negation of " $\rightarrow$ ".

Let $\mathcal{A}_{k} \subset \mathcal{M}$ denote the set of messages for the $k$ th relay. The collection of $\mathcal{A}_{k}$ 's forms a disjoint partitioning of $\mathcal{M}$. Since the source transmits $m_{0}$, we have $\mathcal{A}_{0}=\left\{m_{0}\right\}$. Note that a message and its parities cannot be in the same message set, i.e., $\forall m \neq m^{\prime} \in \mathcal{A}_{k}, m \nrightarrow m^{\prime}$. Furthermore, for $i<j$ there is no message in $\mathcal{A}_{i}$ which is a descendent of $A_{j}$. This property implies that the message of the first relay can only be a parity of the source message, the second relay's message can only be a parity for the first relay or the source message, the third relay's message can only be a parity of the source, the first relay or the second relay's message, and so on. A message is said to be of order $k$ if it belongs to $\mathcal{A}_{k}$. A relay terminal or a message has a lower order if it is assigned to a set $\mathcal{A}_{k}$ with a smaller $k$.

The $k$ th relay encodes $\left\{m_{k 1}^{t}, m_{k 2}^{t}, \cdots, m_{k n_{k}}^{t}\right\}$ in block $t$. The messages sent in block $t$ are parity functions of the messages sent in blocks $t-k$ to $t-1$. More precisely, if $m_{k i}$ is a direct parity of $m_{l j}$, then in block $t$ we have:

$$
m_{k i}^{t}=P_{\mathcal{B}_{k i}}\left(m_{l j}^{t-(k-l)}\right) .
$$

The $k$ th relay must decode a set of messages $\mathcal{D}_{k}$ in order to generate parity messages assigned by $\mathcal{A}_{k}$. The set $\mathcal{D}_{k}$ in general can be any set of messages for which $\mathcal{D}_{k} \rightarrow \mathcal{A}_{k}$. Having decoded the messages in $\mathcal{D}_{k}$, the $k$ th relay knows all messages that are directly or indirectly parities of messages in $\mathcal{D}_{k}$, some of which are assigned to other relay terminals to be transmitted in subsequent blocks. For optimal encoding, the $k$ th relay should cooperate with other relay terminals to transmit messages known to the $k$ th relay.

The $\mathcal{D}_{k}$ sets should have the following properties. First, the set $\mathcal{A}_{k}$ is generated from $\mathcal{D}_{k}$, i.e., $\mathcal{D}_{k} \rightarrow \mathcal{A}_{k}$ while $\mathcal{D}_{k} \cap \mathcal{A}_{k}=\{\}$. Second, if a message $m_{l i}$ is a descendent of $\mathcal{D}_{k}$, then $\forall i^{\prime} \leq i: \mathcal{D}_{k} \rightarrow m_{l i^{\prime}}$ (note that if $m_{r s} \in \mathcal{D}_{k}$ then $\left.\mathcal{D}_{k} \rightarrow m_{r s}\right)$. This property is required, since in superposition broadcast encoding, messages are superimposed on top of each other. In particular, the messages are superimposed in the order of their second subscript; thus, if the $k$ th relay knows a message $m_{l i}$ of the $l$ th relay, then it also knows all other messages of the $l$ th relay with a second subscript less than $i$. Third, a message and its parity cannot be both in $\mathcal{D}_{k}$, i.e., $\forall m \neq m^{\prime} \in \mathcal{D}_{k}: m \nrightarrow m^{\prime}$. Finally, for the destination, we have $\mathcal{D}_{K+1}=\left\{m_{0}\right\}$. Note that a collection of $\mathcal{D}_{k}$ 's always exists, since $\mathcal{D}_{k}=\left\{m_{0}\right\}, 1 \leq k \leq K+1$, satisfies the above properties. 


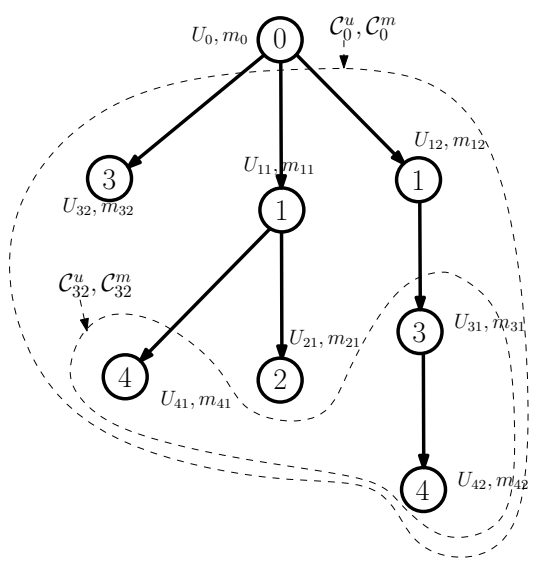

Fig. 2. Examples of known sets for the four-relay network in Fig. 1.

In summary, a parity forwarding protocol for a network with $K$ relay terminals is defined by a three tuple $(\tau, \mathcal{A}, \mathcal{D})$ consisting of the message tree $\tau$, defining the relation between messages and parities; the partitioning $\mathcal{A}$, which assigns the messages to different relay terminals; and the set of decoding sets $\mathcal{D}=\left\{\mathcal{D}_{1}, \mathcal{D}_{2}, \ldots, \mathcal{D}_{K+1}\right\}$, which determines the set of messages each relay should decode.

\section{A. Encoding}

In the parity forwarding protocol, each relay should transmit multiple messages using superposition broadcast encoding. For each message $m_{k l}$, let us first identify the set of all messages on top of which $m_{k l}$ should be encoded.

Definition 2 (Known Sets): First, at the $k$ th relay terminal, the message $m_{k i}$ is encoded on top of all messages $m_{k j}, j<i$. Furthermore, in each block, the $k$ th relay knows all descendent messages of $\mathcal{D}_{k}$ transmitted by relay terminals of orders higher than $k$, i.e., $\left\{m_{k^{\prime} i^{\prime}} \in \mathcal{M}, k^{\prime}>k \mid \mathcal{D}_{k} \rightarrow m_{k^{\prime} i^{\prime}}\right\}$. Hence, a message $m_{k i}$ should be encoded on top of its known set of messages $\mathcal{C}_{k i}^{m}=\left\{m_{k j}, j<i\right\} \cup\left\{m_{k^{\prime} i^{\prime}} \in \mathcal{M} \mid k^{\prime}>k, \mathcal{D}_{k} \rightarrow\right.$ $\left.m_{k^{\prime} l^{\prime}}\right\}$. The known set for the source message consists of all parity messages of the message tree. Let $\mathcal{C}_{k i}^{m}(t)$ denote the instance of $\mathcal{C}_{k i}^{m}$ messages in the $t$ th block. (See Fig. 2 as an example.)

The first step in the generation of random codebooks is to assign a probability distribution and a random variable to each message on the tree. Let $U_{k i}$ represent the random variable corresponding to the encoding of $m_{k i}{ }^{1}$. The set $\mathcal{C}_{k i}^{u}$ is defined as the set of random variables corresponding to messages in $\mathcal{C}_{k i}^{m}$, i.e., $\mathcal{C}_{k i}^{u}=\left\{U_{k i} \mid m_{k i} \in \mathcal{C}_{k i}^{m}\right\}$. The probability distribution $p\left(u_{k i} \mid \mathcal{C}_{k i}^{u}\right)$ is associated with $U_{k i}$. Note that by properties of the decoding sets, $\prod_{k=0}^{K} \prod_{i=1}^{n_{k}} p\left(u_{k i} \mid \mathcal{C}_{k i}\right)$ is a valid joint probability distribution.

To generate random codebooks, we start with messages with empty known sets associated with unconditional probability distributions. For every message $m_{k i}, 1 \leq k \leq K, 1 \leq i \leq$ $n_{i}$ with $\mathcal{C}_{k i}^{m}=\{\}, 2^{n R_{k i}}$ codewords $\mathbf{u}_{k i}\left(m_{k i}\right)$ are randomly

\footnotetext{
${ }^{1}$ For notational simplicity in referring to the source terminal, let us define the 00 subscript to be equivalent to 0 , e.g., $U_{00}$ and $u_{00}$ are equivalent to $U_{0}$ and $u_{0}$, respectively.
}

generated according to probability distribution $p\left(u_{k i}\right)$. In the next step, for every message $m_{k^{\prime} i^{\prime}}$ for which the codewords for all messages in the corresponding $\mathcal{C}_{k^{\prime} i^{\prime}}^{m}$ have already been constructed (in the previous step), $2^{n R_{k^{\prime}} i^{\prime}}$ random codewords are generated for every combination of codewords in $\mathcal{C}_{k^{\prime} i^{\prime}}^{m}$ according to $p\left(u_{k^{\prime} i^{\prime}} \mid \mathcal{C}_{k^{\prime} i^{\prime}}^{u}\right)$ (similar to the code construction for a degraded broadcast channel in which the source encodes messages $m_{k^{\prime} i^{\prime}}$ and $\mathcal{C}_{k^{\prime} i^{\prime}}^{m}$ ). This procedure is repeated until random codebooks are generated for all messages. In block $t$, the $k$ th terminal, $0 \leq k \leq K$, transmits $\mathbf{u}_{k n_{k}}\left(m_{k n_{k}}^{t} \mid \mathcal{C}_{k n_{k}}^{m}(t)\right)$.

\section{B. Decoding}

Let $Y_{1}, Y_{2}, \ldots, Y_{K+1}$ and $\mathbf{y}_{1}^{t}, \mathbf{y}_{2}^{t}, \ldots, \mathbf{y}_{d}^{t}$ denote the random variables and random sequences representing the channel outputs and received sequences in block $t$ at the first relay, the second relay, .... up to the destination, respectively.

At the $k$ th relay terminal, the decoding is performed over a window of successive blocks. Let $\mathcal{A}_{q}$ be the lowest order message set (smallest $q, 0 \leq q \leq K$,) which has an element in $\mathcal{D}_{k}$, i.e., $\mathcal{A}_{q} \cap \mathcal{D}_{k} \neq\{\}$ and $\forall q^{\prime}<q, \mathcal{A}_{q^{\prime}} \cap \mathcal{D}_{k}=\{\}$. Then, according to (1), the decoding window for the $k$ th relay in block $t$ is given by $\left(\mathbf{y}_{k}^{t-(k-q)+1}, \ldots, \mathbf{y}_{k}^{t}\right)$.

Let us identify all parity messages for the messages in $\mathcal{D}_{k}$ available to the $k$ th relay terminal. Any descendent message of $\mathcal{D}_{k}$ sent in or before block $t$ can be used in block $t$ as a parity for the messages in $\mathcal{D}_{k}$. The set of all such messages is defined by $\mathcal{T}_{k}=\left\{m_{r s} \in \mathcal{M} \mid \mathcal{D}_{k} \rightarrow m_{r s}, r<k\right\}$. Note that according to (1), a parity message $m_{r s}$ for $\mathcal{D}_{k}$ with $r \geq k$ is available only after block $t$.

Consequently, the $k$ th terminal, $1 \leq k \leq K+1$, should jointly decode the set of messages $\mathcal{T}_{k}$ in block $t$. The total number of all valid combination of candidate codewords corresponding to messages in $\mathcal{T}_{k}$ is given by

$$
2^{n R_{k}} \triangleq 2^{n\left(\sum_{\forall r, s: m_{r s} \in \mathcal{D}_{k}} R_{r s}\right)}
$$

since the messages in $\mathcal{D}_{k}$ uniquely determine the messages in $\mathcal{T}_{k}$. (For the destination, $\mathcal{T}_{K+1}=\mathcal{M}$, and the total number of all possible candidate codewords is given by the source rate $2^{n R_{0}}$.)

To decode the messages in $\mathcal{T}_{k}$, note that the probability that a codeword $\mathbf{u}_{l j}\left(m_{l j} \mid \mathcal{C}_{l j}^{m}(t-(k-l)+1)\right), q \leq$ $l<k$, generated according to $p\left(u_{l j} \mid \mathcal{C}_{l j}^{u}(t-(k-l)+1)\right)$, is incorrectly declared jointly typical with $\mathbf{y}_{k}^{t-(k-l)}$, given $\mathcal{C}_{l j}^{\mathbf{u}}(t-(k-l)+1)$, is equal to $2^{-n I\left(U_{l j} ; Y_{k} \mid \mathcal{C}_{l j}^{u}\right)}$, where $\mathcal{C}_{l j}^{\mathbf{u}}(t-(k-l)+1)$ denotes the set of codewords corresponding to messages in $\mathcal{C}_{l j}^{m}(t-(k-1)+1)$.

The joint decoding of $\mathcal{T}_{k}$ is successful if a corresponding rate constraint is met for the decoding of all subsets of $\mathcal{T}_{k}$. Let $\mathcal{I}$ be any subset of $\mathcal{T}_{k}$ and $\mathcal{I}^{\prime}=\left\{m_{l i} \in \mathcal{T}_{k} \mid \mathcal{I} \nrightarrow m_{l i}\right\}$. Fixing the messages in $\mathcal{I}$, the probability of error for decoding $\mathcal{I}^{\prime}$ is given by

$$
\left|\mathcal{I}^{\prime}\right| 2^{-n \sum_{\forall h j: m_{h j} \in \mathcal{I}^{\prime}} I\left(U_{h j} ; Y_{k} \mid \mathcal{C}_{h j}^{u}\right)}
$$

where $\left|\mathcal{I}^{\prime}\right|$ denotes the number of all valid combination of messages in $\mathcal{I}^{\prime}$. To compute $\left|\mathcal{I}^{\prime}\right|$ note that $\left|\mathcal{I}^{\prime}\right|=\left|\mathcal{T}_{k}\right|-|\mathcal{I}|$. Let $\mathcal{J}_{\mathcal{I}}$ be the minimal subset of $\mathcal{I}$ which generates $\mathcal{I}$, i.e., $\forall \mathcal{F} \subset$ 


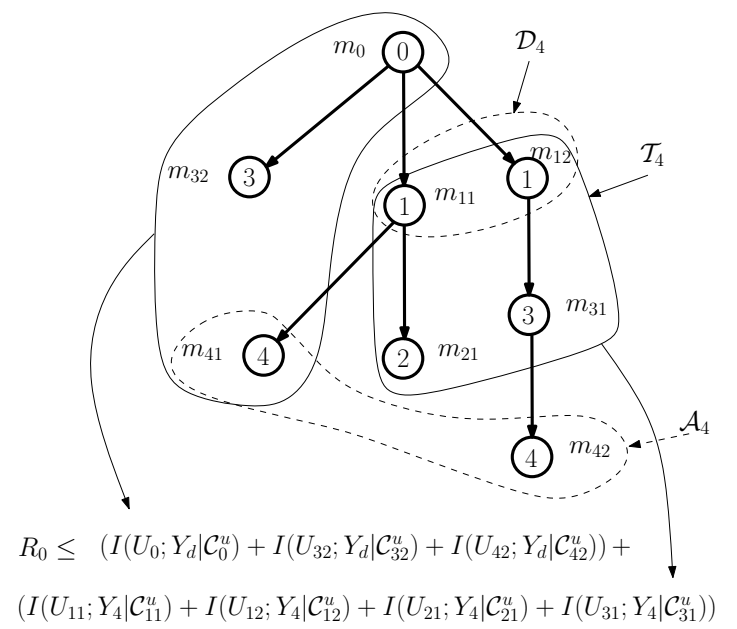

Fig. 3. A description of how the rate constraints are related to the message tree. The above constraint on the source rate is derived by combining the two inequalities derived from (5) by setting $\mathcal{J}_{\mathcal{I}}=\{\}$ for $k=4$, and $\mathcal{J}_{\mathcal{I}}=\mathcal{D}_{4}$ for $k=5$.

$\mathcal{I}: \mathcal{J}_{\mathcal{I}} \rightarrow \mathcal{F}$. Since $\mathcal{J}_{\mathcal{I}}$ generates $\mathcal{I}$, we have $|\mathcal{I}|=\left|\mathcal{J}_{\mathcal{I}}\right|$. Note that $\mathcal{D}_{k}$ is the minimal generator set for $\mathcal{T}_{k}$; hence, we have $\left|\mathcal{T}_{k}\right|=\left|\mathcal{D}_{k}\right|$. Consequently, the decoding error probability at the $k$ th terminal approaches to zero as $n$ goes to infinity if:

$\underbrace{\sum_{\forall h, i: m_{h i} \in \mathcal{D}_{k}} R_{h i}}_{\left|\mathcal{T}_{k}\right|} \leq \sum_{\forall h j: m_{h j} \in \mathcal{I}^{\prime}} I\left(U_{h j} ; Y_{k} \mid \mathcal{C}_{h j}^{u}\right)+\underbrace{\sum_{\forall l, j: m_{l j} \in \mathcal{J}_{\mathcal{I}}} R_{l j}}_{\left|\mathcal{J}_{\mathcal{I}}\right|}$

$\forall \mathcal{I} \subset \mathcal{T}_{k}$.

Summarizing, the following theorem characterizes the achievable rate of the parity forwarding protocol defined by $(\tau, \mathcal{A}, \mathcal{D})$.

Theorem 1 (Achievable Rate of Parity Forwarding):

Consider a memoryless relay network with $K$ relay terminals defined by the probability distribution

$$
p\left(y_{1}, y_{2}, \cdots, y_{K}, y_{K+1} \mid u_{0}, u_{1 n_{1}}, u_{2 n_{2}}, \cdots, u_{K n_{K}}\right) .
$$

For the parity forwarding protocol $(\tau, \mathcal{A}, \mathcal{D})$, the source data rate $R_{0}$ satisfying the following constraints maximized over the probability distribution $\prod_{\forall l, i: m_{l i} \in \mathcal{M}} p\left(u_{l i} \mid \mathcal{C}_{l i}^{u}\right)$ (along with a set of positive rates $R_{h i}$ corresponding to the messages $m_{h i}$ in decoding sets $\left.\mathcal{D}_{k}, 1 \leq k \leq K+1\right)$ is achievable:

$$
\sum_{\forall h, i: m_{h i} \in \mathcal{D}_{k}} R_{h i} \leq \sum_{\forall h j: m_{h j} \in \mathcal{I}^{\prime}} I\left(U_{h j} ; Y_{k} \mid \mathcal{C}_{h j}^{u}\right)+\sum_{\forall l, j: m_{l j} \in \mathcal{J}_{\mathcal{I}}} R_{l j}
$$$$
\forall \mathcal{I} \subset \mathcal{T}_{k}, \forall k: 1 \leq k \leq K+1 \text {. (5) }
$$

It should be noted that some of the inequalities in (5) may be redundant, allowing for further simplification of (5). In particular, an inequality in which there exists an $R_{l j}$ on the right-hand side that does not appear on the left-hand side of another inequality may be ignored, since such an $R_{l j}$ is unbounded.

In the following section we describe simplified examples of parity forwarding protocols for chain networks. New forms of degraded multirelay networks for which parity forwarding is capacity achieving are also identified.

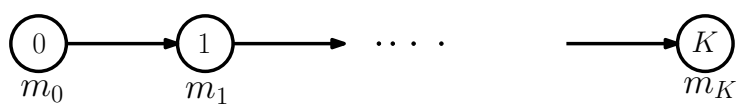

Fig. 4. Chain message tree.

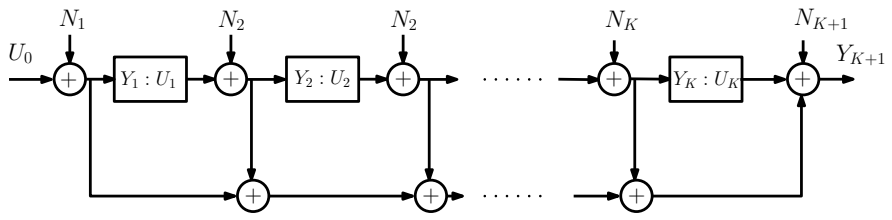

Fig. 5. A degraded chain network with additive noises $N_{k}, 1 \leq k \leq K$. Each relay can directly communicate only to its successor terminal. The $k$ th relay decodes the message of relay $k-1$ and forwards a parity for it.

\section{Examples with Chain Message Tree}

Consider a relay network with $K$ relay terminals ordered from 1 to $K$. The message tree shown in Fig. 4 corresponds to parity forwarding protocols in which the $k$ th relay transmits a parity message for the message of relay $k-1$.

For a given network, various parity forwarding protocols can be devised corresponding to different message trees and different choices of message sets and decoding sets. For example, for the chain message tree shown in Fig. 4, each relay terminal may only decode the message of its predecessor. This would be a good scheme if each relay terminal has a small range and can only communicate to its successor relay (e.g., the degraded network shown in Fig. 5). Such a scheme corresponds to setting $\mathcal{D}_{k}=\left\{m_{k-1}\right\}, 1 \leq k \leq K$, and $\mathcal{D}_{K+1}=\left\{m_{0}\right\}$. Consequently, we have $\mathcal{C}_{k}^{m}=\left\{m_{k+1}, m_{k+2}, \cdots, m_{K}\right\}, 0 \leq$ $k \leq K, \mathcal{T}_{k}=\left\{m_{k-1}\right\}, 0<k \leq K, 1<k \leq K$, and $\mathcal{T}_{K+1}=\left\{m_{0}, m_{1}, \cdots, m_{K}\right\}$.

The achievable rate given by Theorem 1 for this protocol can be computed as follows. For $1 \leq k \leq K$, the only subsets $\mathcal{I}$ of $\mathcal{T}_{k}=\left\{m_{k-1}\right\}$ are \{\} and $\mathcal{T}_{k}$. Hence, the inequalities corresponding to $k=1$ in (5) are given by

$R_{0} \leq I\left(U_{0} ; Y_{1} \mid U_{1}, U_{2}, \cdots, U_{K}\right) \quad: \mathcal{I}=\{\}, \mathcal{I}^{\prime}=\mathcal{T}_{1}=\left\{m_{0}\right\}$

$R_{0} \leq R_{0} \quad: \mathcal{I}=\left\{m_{0}\right\}, \mathcal{I}^{\prime}=\{\}$

Similarly, for $2 \leq k \leq K$, (5) requires that

$\begin{array}{ll}R_{k-1} \leq I\left(U_{k-1} ; Y_{k} \mid U_{k}, \cdots, U_{K}\right) & : \mathcal{I}=\{\}, \mathcal{I}^{\prime}=\left\{m_{k-1}\right\} \\ R_{k-1} \leq R_{k-1} & : \mathcal{I}=\left\{m_{k-1}\right\}, \mathcal{I}^{\prime}=\{\}\end{array}$

Finally, since $\mathcal{T}_{K+1}=\left\{m_{0}, m_{1}, \cdots, m_{K}\right\}$ and $\mathcal{D}_{K+1}=$ $\left\{m_{0}\right\}$, (5) gives the following inequalities for $k=K+1$ :

$$
R_{0} \leq \sum_{i=1}^{l-1} I\left(U_{i} ; Y_{K+1} \mid U_{i+1}, \cdots, U_{K+1}\right)+R_{l}, 2 \leq l \leq K
$$

The above inequalities are derived by setting $\mathcal{J}_{\mathcal{I}}=\left\{m_{l}\right\}$ and $\mathcal{I}^{\prime}=\left\{m_{0}, \cdots, m_{l-1}\right\}$ for $2 \leq l \leq K$.

Using the chain rule for the mutual information and substituting the constraints on $R_{l}$ for $2 \leq l \leq K$ in (6) results in 

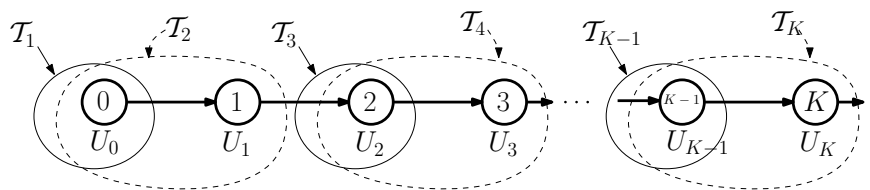

Fig. 6. The sets $\mathcal{T}_{k}$ for the example in Fig. 7.

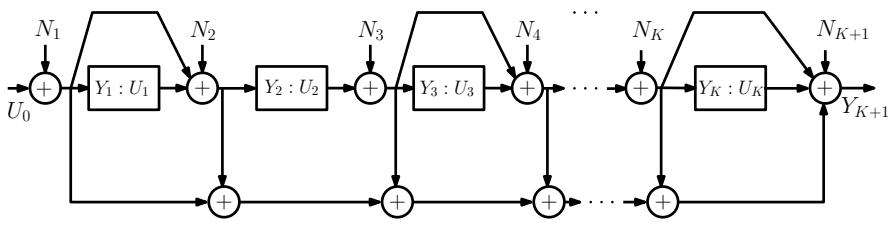

Fig. 7. A degraded chain network with additive noise. For $k$ even, the $k$ th relays can only communicate to its direct successor. For odd $k$ 's, the $k$ th relay helps the $(k+1)$ th relay decode the message of the $(k-1)$ th relay.

the following achievable rate:

$$
\begin{aligned}
& R_{0}<I\left(U_{0} ; Y_{1} \mid U_{1}, U_{2}, \ldots, U_{K}\right) \\
& R_{0}<I\left(U_{1} ; Y_{2} \mid U_{2}, \ldots, U_{K}\right)+I\left(U_{0} ; Y_{K+1} \mid U_{1}, \ldots, U_{K}\right)+ \\
& R_{0}<I\left(U_{2} ; Y_{3} \mid U_{3}, \ldots, U_{K}\right)+I\left(U_{0}, U_{1} ; Y_{K+1} \mid U_{2}, \ldots, U_{K}\right) \\
& \quad \vdots \\
& \quad \\
& R_{0}<I\left(U_{K-1} ; Y_{K} \mid U_{K}\right)+I\left(U_{0}, \ldots, U_{K-2} ; Y_{K+1} \mid U_{K-1}, U_{K}\right) \\
& R_{0}<I\left(U_{0}, U_{1}, \ldots, U_{K} ; Y_{K+1}\right) .
\end{aligned}
$$

Using the cut-set bound [8, Theorem 14.10.1], it can be proved that this rate is indeed the capacity for the degraded network shown in Fig. 5. This is intuitive since, each rateconstraint in the above achievable rate consists of two components: one component represents the transferable information from a relay terminal to its successor, and the other component corresponds to the cooperative transferable information from all lower order relays to the destination. This coincides with the rate given by the cut-set defined at the $k$ th relay separating the source and the first $k$ relays from the destination the rest of the relays.

As another example, consider the protocol described by the message tree depicted in Fig. 6. In this network, the source and the first relay cooperate to communicate to the second relay. The second relay and the third relay cooperate to communicate to the fourth relay, and so on. Assume that $K$ is odd. The $k$ th relay decodes the message of relay $k-1$ for odd $k$ 's, and decodes the message of relay $k-2$ for even $k$ 's. This scenario corresponds to setting $\mathcal{D}_{k}=\left\{m_{k-1}\right\}$ for odd $k$ 's, and $\mathcal{D}_{k}=\left\{m_{k-2}\right\}$ for even $k$ 's. Hence, $\mathcal{T}_{k}=\left\{m_{k-1}\right\}$ for odd $k$ 's, and $\mathcal{T}_{k}=\left\{m_{k-2}, m_{k-1}\right\}$ for even $k$ 's (Fig. 6). For the destination, $\mathcal{T}_{K+1}=\left\{m_{0}, \cdots, m_{K}\right\}$, and $D_{K+1}=\left\{m_{0}\right\}$.

Using the same approach as in the previous example, the following rate is achievable by Theorem 1:

$$
\begin{aligned}
& R_{0}<I\left(U_{0} ; Y_{1} \mid U_{1}, \cdots, U_{K}\right) \\
& R_{0}<I\left(U_{0}, U_{1} ; Y_{2} \mid U_{2}, \cdots, U_{K}\right) \\
& R_{0}<I\left(U_{2} ; Y_{3} \mid U_{3}, \cdots, U_{K}\right)+I\left(U_{0}, U_{1} ; Y_{K+1} \mid U_{2}, \cdots, U_{K}\right) \\
& R_{0}<I\left(U_{2}, U_{3} ; Y_{4} \mid U_{4}, \cdots, U_{K}\right)+I\left(U_{0}, U_{1} ; Y_{K+1} \mid U_{2}, \cdots, U_{K}\right)
\end{aligned}
$$

$$
\begin{gathered}
R_{0}<I\left(U_{K-2} ; Y_{K-1} \mid U_{K-1, U_{K}}\right)+ \\
I\left(U_{0}, U_{1}, \cdots, U_{K-3} ; Y_{K+1} \mid U_{K-2}, \cdots, U_{K}\right) \\
R_{0}<I\left(U_{K-2}, U_{K-1} ; Y_{K} \mid U_{K}\right)+ \\
\quad I\left(U_{0}, U_{1}, \cdots, U_{K-3} ; Y_{K+1} \mid U_{K-2}, U_{K-1}, U_{K}\right) \\
R_{0}<I\left(U_{0}, U_{1}, \cdots, U_{K} ; Y_{K+1}\right)
\end{gathered}
$$

The above rate meets the cut-set bound for the degraded network shown in Fig. 7. This is intuitively justified if we consider the cut-set defined at the $k$ th relay, separating the source and the first $k$ relays from the destination and relays $k+1$ up to $K$. For an odd $k$, the rate of this cut-set equals to the cooperative information rate from relays $k-1$ and $k$ to relay $k+1$, plus the cooperative rate from the source and relays 1 up to $k-2$ to the destination. For an even $k$, the rate of this cut-set is given by the rate at which the $k$ th terminal can communicate to relay $k+1$, plus the rate at which the source and the first $k-1$ relays can communicate to the destination.

Finally, we note that the previous best multihop DF rate derived in [1] is achieved by setting $\mathcal{D}_{k}=\left\{m_{0}\right\}, 1 \leq k \leq$ $K+1$. The DF rates proposed in this paper are more general and they improve that of [1].

\section{CONClusions}

This paper formulates a class of DF strategies for an arbitrary multirelay network. In this set of strategies, called parity forwarding, relay nodes forward bin indices for the messages of other transmitters. The message tree structure is utilized to characterize the encoding and decoding procedures and the dependencies between messages and their bin indices. Parity forwarding improves previous DF strategies because of its flexibility, and achieves the capacity of new forms of degraded multirelay networks. To derive closed-form expressions for the achievable rate, we restricted ourselves to the superposition encoding strategy of the degraded broadcast channel; further generalization is viable by using more sophisticated broadcasting schemes.

\section{REFERENCES}

[1] L. L. Xie and P. R. Kumar, "An achievable rate for the multiple-level relay channel," IEEE Trans. Inform. Theory, vol. 51, no. 4, pp. 1348-1358, Apr. 2005.

[2] G. Kramer, M. Gastpar, and P. Gupta, "Cooperative strategies and capacity theorems for relay networks," IEEE Trans. Inform. Theory, vol. 51, no. 9, pp. 3037-3063, Sept. 2005.

[3] P. Gupta and P. R. Kumar, "Towards an information theory of large networks: an achievable rate region," IEEE Trans. Inform. Theory, vol. 49, no. 8, pp. 1877-1894, Aug. 2003.

[4] A. Reznik, S. R. Kulkarni, and S. Verdu, "Degraded Gaussian multirelay channel: capacity and optimal power allocation," IEEE Trans. Inform. Theory, vol. 50, no. 12, pp. 3037-3046, Dec. 2004.

[5] M. Gastpar and M. Vetterli, "On the capacity of large Gaussian relay networks," IEEE Trans. Inform. Theory, vol. 51, no. 3, pp. 765-779, March 2005.

[6] T. M. Cover and A. A. El Gamal, "Capacity theorems for the relay channel," IEEE Trans. Inform. Theory, vol. 25, no. 5, pp. 572-584, Sept. 1979.

[7] P. Razaghi and W. Yu, "Parity forwarding for multiple-relay networks," in Proc. of IEEE Int. Symp. Inform. Theory, 2006, pp. 1678-1682.

[8] T. M. Cover and J. A. Thomas, Elements of Information Theory, John Wiley and Sons, 1991. 